\title{
High Intrachain Hole Mobility on Molecular Wires of Ladder-Type Poly( $p$-Phenylenes)
}

\author{
P. Prins, ${ }^{1}$ F. C. Grozema, ${ }^{1}$ J. M. Schins,${ }^{1}$ S. Patil,${ }^{2}$ U. Scherf, ${ }^{2}$ and L. D. A. Siebbeles ${ }^{1}$ \\ ${ }^{1}$ Opto-Electronic Materials Section, DelftChemTech, Delft University of Technology, Mekelweg 15, 2629 JB Delft, The Netherlands \\ ${ }^{2}$ Department of Chemistry and Institute for Polymer Technology, Bergische Universität Wuppertal, \\ Gaußstraße 20, 42119, Wuppertal, Germany \\ (Received 9 December 2005; published 13 April 2006)
}

\begin{abstract}
We have studied the high-frequency $(34 \mathrm{GHz}$ ) mobility of positive charge carriers on isolated laddertype polymer chains in dilute solution. We find that the high-frequency mobility is limited by the chain ends on chains as long as 35 monomers. The intrachain motion of charge carriers can be described by onedimensional diffusion between infinitely high reflecting barriers, representing the chain ends. Our data indicate that the intrachain mobility for ladder-type polymer chains is close to $600 \mathrm{~cm}^{2} / \mathrm{V} \mathrm{s}$. With this high mobility the ladder-type polymer is a promising candidate for future use as an interconnecting wire in molecular electronics.
\end{abstract}

The optoelectronic properties of conjugated polymers are of interest due to their applications in devices, such as organic light-emitting diodes, photovoltaic cells, and field-effect transistors [1-3]. Using solution-processable polymers as semiconducting compounds opens the way to flexible electronics and enables cheap and easy production of devices [4,5]. Moreover, the ongoing miniaturization of electronic components rapidly approaches the fundamental limit of photolithography. Therefore it is of interest to study the ultimate size limit for organic electronics. Single molecules are the smallest components that are likely to be functional in integrated circuits [68]. Conjugated polymers can be used as interconnecting molecular wires between molecular scale electronic components.

In all applications, the mobility of charge carriers is a key factor determining the performance of the electronic devices. Recently, charge carrier mobilities as high as 15 to $35 \mathrm{~cm}^{2} / \mathrm{V}$ s have been found for organic oligoacene crystals $[9,10]$. In these materials the calculated bandwidth is of the order of a few tenths of an electron volt $[11,12]$. For perfectly ordered conjugated polymer chains the bandwidth is of the order of a few electron volts [13]. As a consequence the mobility of charge carriers moving along ordered conjugated polymer chains is expected to be considerably higher than the mobilities found for oligoacene crystals, i.e., on the order of a few hundred $\mathrm{cm}^{2} / \mathrm{V} \mathrm{s}$. The values found experimentally for the device mobility in conjugated polymers however, range from $10^{-7}$ to $10^{-1} \mathrm{~cm}^{2} / \mathrm{V} \mathrm{s}$, for derivatives of poly(para-phenylenvinylene), poly(thienylenevinylene), and poly(thiophene) $[3,14,15]$, which is several orders of magnitude lower than expected.

This discrepancy is likely to be due to a combination of device-specific properties and intrinsic material properties. Contact effects are the most important of the devicespecific properties, and determine the performance of organic optoelectronic devices to a large extent $[16,17]$.
Intrinsic properties of organic materials that limit the charge carrier mobility are mostly related to disorder in the material. Two examples are interchain transport and charge transport over grain boundaries (boundary junctions between different relatively ordered domains within the sample). Both processes are expected to be significantly slower than intrachain transport and depend strongly on the order on a supramolecular scale. An important factor that reduces the intrachain mobility in conjugated polymers is the limited conjugation length of the polymer. Such a reduction of the conjugation length can be caused by (torsional) disorder along the polymer backbone or the presence of chemical defects that lead to barriers to charge transport [18-20].

In this Letter we show that the intrachain mobility for charge carriers moving along ordered polymer chains can be considerably higher than the mobilities found for oligoacene crystals, i.e., on the order of a few hundred $\mathrm{cm}^{2} / \mathrm{V} \mathrm{s}$. To obtain insight into the intrachain motion of charge carriers and to circumvent device-specific effects and effects that result from interchain disorder, we have studied the motion of charges along isolated polymer chains. To gain understanding about the effects of barriers to charge transport, we have systematically varied the chain length of the conjugated backbone. The polymers studied are the ribbonlike ladder-type poly ( $p$-phenylenes) shown in the inset of Fig. 1 [21]. The polymer chains are restricted to a planar geometry by a bridging carbon atom. Hence, the conjugation length is not limited by geometrical distortions. The experiments discussed here were performed on dilute solutions of the ladder-type polymer in benzene. In this way the polymer chains were isolated from each other. Charge carriers were generated by irradiation of the dilute polymer solution with a $10 \mathrm{~ns}$ pulse of $3 \mathrm{MeV}$ electrons from a Van de Graaff electron accelerator. The high-energy electrons scatter on the solvent molecules and produce a close to uniform distribution of excess electrons and benzene cations with a known concentration. These 


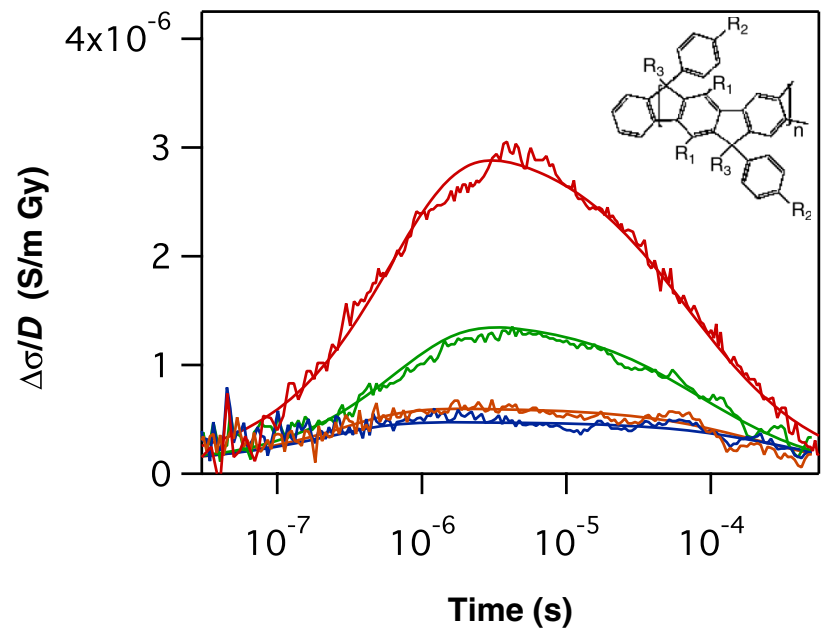

FIG. 1 (color online). Conductivity due to positive charges on ladder-type polymers with a number averaged chain length of 13 , 16,35 , and 54 repeat units, from bottom to top. The measurements were performed at a monomer concentration of $0.315 \mathrm{mM}$, a microwave frequency of $34 \mathrm{GHz}$, an electric field strength of $20 \mathrm{~V} / \mathrm{cm}$, and an irradiation dose $(D)$ of $21 \mathrm{~Gy}$. The inset shows the chemical structure of the ladder-type polymers used in this study. $\quad R_{1}=n$-hexyl, $\langle n\rangle=13,16,35: \quad R_{2}=t$-butyl, $\quad R_{3}=$ phenyl, $\langle n\rangle=54: R_{2}=n$-decyl, $R_{3}=C$.

excess electrons and benzene cations can diffuse towards the polymer chains, where they undergo charge transfer, thus yielding a charge on the polymer backbone [22]. The change in conductivity after the generation of charges was monitored by time resolved microwave conductivity (TRMC) measurements [23]. With this technique, the absorbance of microwave power as a result of the presence of (mobile) charges is monitored. With the TRMC method, we are able to determine the high-frequency mobility of charges on isolated polymer chains in solution, without the use of electrodes [18,22,24,25].

In Fig. 1 we present the change in conductivity after the generation of charge carriers for the ladder-type polymers shown in the inset. In order to selectively study the mobility of holes along the polymer chains, the solution was saturated with oxygen. Because of the relatively high concentration $(12 \mathrm{mM})$ and electron affinity of oxygen, the excess electrons generated during the electron pulse $\left(e_{b z}{ }^{-}\right)$rapidly react with the oxygen molecules $\left(\mathrm{O}_{2}\right)$ forming the oxygen anion $\left(\mathrm{O}_{2}{ }^{-}\right)$:

$$
e_{b z}{ }^{-}+\mathrm{O}_{2} \rightarrow \mathrm{O}_{2}{ }^{-} \text {. }
$$

In this way, the transfer of negative charges to the polymer chains is prevented. Since the mobility of the oxygen anion in benzene is low $\left(10^{-3} \mathrm{~cm}^{2} / \mathrm{V} \mathrm{s}\right)$ [26] as compared to the mobility of the positive charges along the polymer chain (see below), the contribution of the oxygen anion to the observed change in conductivity is negligible. The benzene cations generated during the $10 \mathrm{~ns}$ electron pulse $\left(b z^{+}\right)$ react with the ladder-type polymer (LPPP) by a diffusion controlled reaction, yielding positively charged polymer chains $\left(\mathrm{LPPP}^{+}\right)$

$$
b z^{+}+\mathrm{LPPP} \rightarrow \mathrm{LPPP}^{+}+b z .
$$

As this reaction proceeds, an increase in the transient conductivity is observed on a time scale of hundreds of nanoseconds. This increase directly indicates that the positive charge on the polymer chain is more mobile than the benzene cation in benzene solution $\left(1.2 \times 10^{-3} \mathrm{~cm}^{2} / \mathrm{V} \mathrm{s}\right.$ [27]). On a time scale of ten to hundreds of microseconds a decrease in the conductivity signal is observed. The time scale of this decay decreases with increasing initial concentration of charge carriers, and can therefore be attributed to charge recombination between the oxygen anion and the positive charge on the polymer chain

$$
\mathrm{O}_{2}{ }^{-}+\mathrm{LPPP}^{+} \rightarrow \mathrm{LPPP}+\mathrm{O}_{2} \text {. }
$$

A more extensive description of the reactions upon irradiation with high-energy electrons and the resulting transient conductivity of dilute polymer solutions can be found elsewhere [22,24].

To obtain the high-frequency (ac) mobility for holes $\left(\mu_{\text {ac,hole }}\right)$ from the measured conductivity shown in Fig. 1, the time dependent concentrations $\left[n_{i}(t)\right]$ of charged species $i$ must be known. These concentrations are obtained from a kinetic analysis of the conductivity transients, involving reactions (1)-(3) [22]. The highfrequency mobility of holes on the polymer chains can now be obtained by using

$$
\Delta \sigma(t)=e \sum_{i} \mu_{\mathrm{ac}, i} n_{i}(t),
$$

where $e$ is the elementary charge and $\mu_{\mathrm{ac}, i}$ denotes the high-frequency mobility of charged species $i$. The results for the conductivity as a function of time calculated according to Eq. (4) are shown in Fig. 1 by smooth curves.

As the average chain length increases, the highfrequency hole mobility is found to increase an order of magnitude from 0.025 via 0.036 , and 0.10 to $0.24 \mathrm{~cm}^{2} / \mathrm{V} \mathrm{s}$ for an average chain length of $13,16,35$, and 54 repeat units, respectively. This increase in mobility with chain length is indicative of hindrance of charge carrier motion by the chain ends, at least for chains as long as 35 monomers. The fact that we observe this chain length dependence for the high-frequency mobility in our microwave conductivity measurements, means that the charge must diffuse over the entire length of the polymer chain, and encounter a chain end on a time scale on the order of 1 period of the oscillating electric field. For onedimensional diffusion, the mean squared displacement as a function of time is given by $\left\langle x^{2}(t)\right\rangle=2 D t$, where the diffusion constant $D$ is related to the intrachain mobility of the particle performing diffusive motion $\left(\mu_{\text {intra }}\right)$ and temperature $(T)$ according to the Einstein relation $D=$ $\mu_{\text {intra }} k_{B} T / e$. Using a displacement equal to a chain length of 35 monomers ( $30 \mathrm{~nm}$ ), and a migration time equal to one period of the microwave field ( $30 \mathrm{ps}$ ), a value of $5 \mathrm{~cm}^{2} / \mathrm{V} \mathrm{s}$ 
can be deduced as a lower limit to the intrachain mobility for positive charges moving along ladder-type polymers.

In the limit of a small (probing) electric field, the diffusive motion of a charge determines the frequency dependence of the mobility as has been shown by Kubo [28]. Therefore, we can calculate the high-frequency mobility of charges along polymer chains by using a model that describes the diffusive motion of the charge. The polymer chain is modeled by a one-dimensional flat energy surface between infinitely high reflecting barriers. Comparison of the experimental results for different average polymer chain lengths with the mobilities calculated using this model gives the absolute value of the intrachain mobility.

In the one-dimensional diffusion model the highfrequency mobility of the charge carriers $\left(\mu_{\mathrm{ac}}\right)$ depends on the (radial) frequency $(\omega)$, the length of the polymer chain $(a)$, and the intrachain mobility $\left(\mu_{\text {intra }}\right)$. The highfrequency mobility of charges can be expressed in terms of the intrachain diffusion coefficient $(D)$ according to

$$
\mu_{\mathrm{ac}}=8 D \frac{e}{k_{B} T} \sum_{k=0}^{\infty} \frac{\left[c_{k}\right]^{-2}}{\left[\frac{D}{a^{2} \omega}\right]^{2}\left[c_{k}\right]^{4}+1},
$$

where

$$
c_{k}=2 \pi\left(k+\frac{1}{2}\right) .
$$

The details of the derivation and the physical interpretation of Eq. (5) are discussed in the supporting information [29]. To obtain the high-frequency mobility as a function of the intrachain mobility for the average polymer chain lengths used in the experiment, the high-frequency mobility calculated with Eq. (5) is averaged over the chain length according to a truncated Flory distribution with $5 \leq n \leq 75$ [29]. The length of the ladder-type chains $(a)$ is the product of the number of repeat units and the length of one repeat unit $(8.3 \AA)$. The results for the four average chain lengths are shown in Fig. 2(a).

For low intrachain mobility, the high-frequency mobility is equal to the intrachain mobility; the motion of charges is not significantly hindered by the chain ends during the oscillation period of the probing field. The high-frequency mobility starts to deviate from the intrachain mobility when the intrachain mobility exceeds a few tenths of $\mathrm{cm}^{2} / \mathrm{V} \mathrm{s}$, since the charge carrier starts to encounter the ends of the polymer chain. As the intrachain mobility increases to tens of $\mathrm{cm}^{2} / \mathrm{Vs}$, scattering of the charge carriers at the chain ends starts to dominate the charge transport and the high-frequency mobility decreases with increasing intrachain mobility.

In order to describe the experimental data in terms of the one-dimensional diffusion model discussed above, we need to determine the intrachain mobility that gives rise to the experimental values for the high-frequency hole mobilities for the four average chain lengths. Mobilities in the order of the experimental values, denoted by the horizontal lines in Fig. 2, are obtained both at a few tenths of $\mathrm{cm}^{2} / \mathrm{V} \mathrm{s}$ and at a few hundred $\mathrm{cm}^{2} / \mathrm{V} \mathrm{s}$. At low intra-
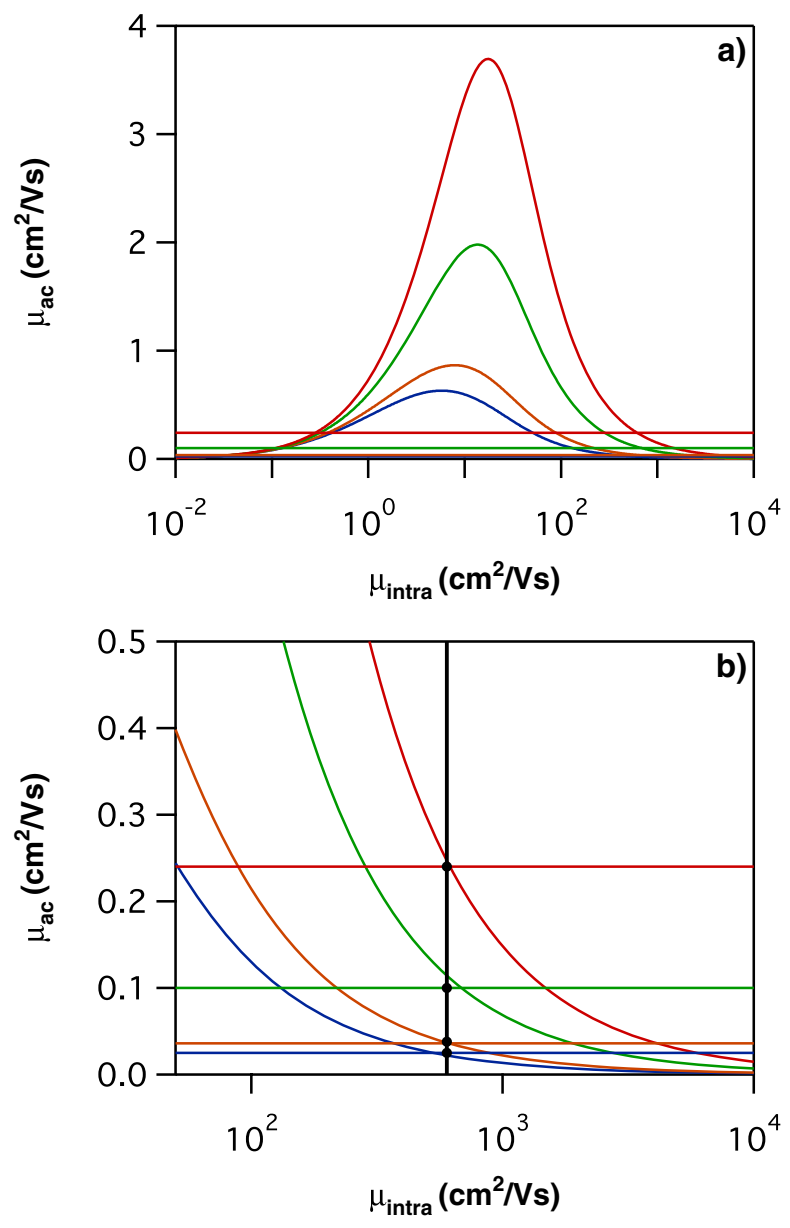

FIG. 2 (color online). (a) High-frequency mobility as a function of intrachain mobility calculated with Eq. (5), for an average polymer chain length of $13,16,35$, and 54 repeat units, from bottom to top. The horizontal lines represent the experimental data. (b) The experimental values for the high-frequency mobility are reproduced by an intrachain mobility close to $600 \mathrm{~cm}^{2} / \mathrm{V} \mathrm{s}$.

chain mobility, almost no chain length dependence would be observed for the high-frequency mobility probed by the microwave field, see Fig. 2(a). Thus, the experimentally observed increase in the high-frequency mobility with chain length cannot be reproduced with an intrachain mobility in the order of a few tenths of $\mathrm{cm}^{2} / \mathrm{Vs}$. This agrees with the estimate made above, that the intrachain mobility must exceed $5 \mathrm{~cm}^{2} / \mathrm{Vs}$ in order to explain the chain length dependence of the experimental results. The data in Fig. 2(b) show that the measured high-frequency mobilities for different average chain length are reproduced by an intrachain mobility close to $600 \mathrm{~cm}^{2} / \mathrm{V} \mathrm{s}$.

For an intrachain mobility of $600 \mathrm{~cm}^{2} / \mathrm{V} \mathrm{s}$ the charge carrier motion along the polymer chains used in this study is severely hindered by the polymer chain ends. Hence, the one-dimensional diffusion model predicts a strong frequency dependence for the high-frequency mobility. Therefore, the high-frequency mobility was determined at a microwave frequency of $10.6 \mathrm{GHz}$ for the ladder- 
type polymer in dilute solution. A high-frequency hole mobility of $0.032 \mathrm{~cm}^{2} / \mathrm{V} \mathrm{s}$ is obtained for the polymer with an average chain length of 54 repeat units. This value is significantly ( 7.5 times) lower than the mobility found for the same polymer at $34 \mathrm{GHz}$. The one-dimensional diffusion model in Eq. (5) gives a high-frequency mobility of $0.031 \mathrm{~cm}^{2} / \mathrm{V} \mathrm{s}$ at a microwave frequency of $10.6 \mathrm{GHz}$ $\left(\langle n\rangle=54, \mu_{\text {intra }}=600 \mathrm{~cm}^{2} / \mathrm{V} \mathrm{s}\right)$ which is in very good agreement with the experimental value. Thus the frequency dependence of the mobility substantiates the value of $600 \mathrm{~cm}^{2} / \mathrm{V} \mathrm{s}$ for the intrachain mobility deduced from the chain length dependence.

The results clearly show that limitations in the conjugation length determine the charge transport properties in conjugated polymers to a large extent. In the ribbonlike ladder-type polymer studied here the chain ends determine the effective conjugation length of the polymer. In other conjugated polymers, however, the effective conjugation is often reduced by defects or (torsional) disorder and thus much shorter than the length of the polymer backbone. Therefore, in addition to device-specific improvements (such as the reduction of injection barriers at the contacts) enhancement of order and reduction of defect concentration are expected to significantly improve the performance of molecular electronic devices. Furthermore, we have shown that the intrachain mobility for conjugated polymers is more than 3 orders of magnitude higher than the interchain mobility determined using devices [30]. This means that one of the major limitations of organic electronics with respect to inorganic electronics, can be overcome. In the ultimate size limit for organic electronics, single molecules will be used both as electronic components and as interconnecting wires between these components. If these wires consist of ladder-type polymer chains, with an intrachain mobility of $600 \mathrm{~cm}^{2} / \mathrm{V} \mathrm{s}$, the device performance will not be limited by the transport of charges between the components. Therefore ladder-type polymers are promising candidates for use in molecular electronics.

This work is part of the research program of the "Stichting voor Fundamenteel Onderzoek der Materie (FOM)," which is financially supported by the "Nederlandse Organisatie voor Wetenschappelijk Onderzoek (NWO)."

[1] L. L. Chua, J. Zaumsell, J. F. Chang, E. C. W. Ou, P. K. H. Ho, H. Sirringhaus, and R.H. Friend, Nature (London) 434, 194 (2005).

[2] H. Sirringhaus, N. Tessler, and R. H. Friend, Science 280, 1741 (1998).

[3] H. E. A. Huitema, G. H. Gelinck, J. B. P. H. van der Putten, K. E. Kuijk, K. M. Hart, E. Cantatore, and D. M. de Leeuw, Adv. Mater. 14, 1201 (2002).

[4] S. P. Speakman, G. G. Raozenberg, K. J. Clay, W. I. Milne, A. Ille, I. A. Gardner, E. Bresler, and J. H. G. Steinke, Org. Electron. 2, 65 (2001).
[5] F. Hide, M. A. Diaz-Garcia, B. J. Schwartz, and A.J. Heeger, Acc. Chem. Res. 30, 430 (1997).

[6] I. Heller, J. Kong, H. A. Heering, K. A. Williams, S. G. Lemay, and C. Dekker, Nano Lett. 5, 137 (2005).

[7] S. Kubatkin, A. Danilov, M. Hjort, J. Cornil, J. Bredas, N. Sturh-Hansen, P. Hedegard, and T. Bjornholm, Curr. Appl. Phys. 4, 554 (2004).

[8] C. Joachim, J.K. Gimzewske, and A. Aviram, Nature (London) 408, 541 (2000).

[9] O.D. Jurchescu, J. Baas, and T. M. Palstra, Appl. Phys. Lett. 84, 3061 (2004).

[10] V.C. Sundar, J. Zaumseil, V. Podzorov, E. Menard, R. Willett, T. Someya, M.E. Gershenson, and J.A. Rogers, Science 303, 1644 (2004).

[11] D. A. da Silva Filho, E. Kim, and J. Bredas, Adv. Mater. 17, 1072 (2005).

[12] Y. C. Cheng, R. J. Silbey, D. A. da Silva Filho, J. P. Calbert, J. Cornil, and J. Bredas, J. Chem. Phys. 118, 3764 (2003).

[13] M. Lögdlund and W. R. Salaneck, in Semiconducting Polymers, edited by G. Hadziioannou and P.F. van Hutten (Wiley-Vch, Weinheim, 2000), p. 115.

[14] P. W. M. Blom, M. J. M. de Jong, and J. J. M. Vleggaar, Appl. Phys. Lett. 68, 3308 (1996).

[15] H. Sirringhaus, P. Brown, R. H. Friend, M. M. Nielsen, K. Bechgaard, B. M. W. Langeveld-Voss, A. J. H. Spiering, R. A. J. Janssen, E. W. Meijer, P. Herwig, and D. M. de Leeuw, Nature (London) 401, 685 (1999).

[16] H. Ishii, K. Sugiyama, E. Ito, and K. Seki, Adv. Mater. 11, 605 (1999).

[17] M. A. Baldo and S. R. Forrest, Phys. Rev. B 64, 085201 (2001).

[18] F. C. Grozema, L. D. A. Siebbeles, J. M. Warman, S. Seki, S. Tagawa, and U. Scherf, Adv. Mater. 14, 228 (2002).

[19] F. C. Grozema, P. T. v. Duijnen, Y. A. Berlin, M. A. Ratner, and L. D. A. Siebbeles, J. Phys. Chem. B 106, 7791 (2002).

[20] L. P. Candeias, F. C. Grozema, G. Padmanaban, S. Ramakrishnan, L. D. A. Siebbeles, and J. M. Warman, J. Phys. Chem. B 107, 1554 (2003).

[21] W. Graupner, M. Mauri, J. Stampfl, G. Leising, U. Scherf, and K. Mullen, Solid State Commun. 91, 7 (1994).

[22] F. C. Grozema, R. J. O. M. Hoofman, L. P. Candeias, M. P. de Haas, J. M. Warman, and L. D. A. Siebbeles, J. Phys. Chem. A 107, 5976 (2003).

[23] P. P. Infelta, M. P. de Haas, and J. Warman, Radiat. Phys. Chem. 10, 353 (1977).

[24] P. Prins, L. P. Candeias, A. J. J. M. van Breemen, J. Sweelssen, P. T. Herwig, H. F. M. Schoo, and L. D. A. Siebbeles, Adv. Mater. 17, 718 (2005).

[25] R. J. O. M. Hoofman, M.P. de Haas, L. D. A. Siebbeles, and J. M. Warman, Nature (London) 392, 54 (1998).

[26] A. O. Allen, M. P. de Haas, and A. Hummel, J. Chem. Phys. 64, 2587 (1976).

[27] S. S. S. Huang and G. R. Freeman, J. Chem. Phys. 72, 1989 (1980).

[28] R. Kubo, J. Phys. Soc. Jpn. 12, 570 (1957).

[29] See EPAPS Document No. E-PRLTAO-96-036616 for supplementary material. For more information on EPAPS, see http://www.aip.org/pubservs/epaps.html.

[30] D. Hertel, U. Scherf, and H. Bässler, Adv. Mater. 10, 1119 (1998). 